\title{
Autoimmune Disease
}

\author{
Tobias Alexander, Basil Sharrack, \\ Montserrat Rovira, Dominique Farge, \\ and John A. Snowden
}

\subsection{Introduction}

Autoimmune diseases (ADs) are a heterogeneous group of diseases affecting 8-10\% of the Western population, which constitute a heavy burden to society and are often debilitating and disabling for affected individuals. Current therapeutic strategies for AD are based on systemic immunosuppression (IS), which ameliorates symptoms and halts progression in the vast majority of patients, but usually require continuous administration and may be associated with long-term side effects and substantial costs. Although introduction of modern biological therapies dramatically improved the treatment landscape in $\mathrm{AD}$, cure remains elusive, and many patients still suffer from progressive disability with shortened life expectancy and comorbidity.

\footnotetext{
T. Alexander $(\bowtie)$

Department of Rheumatology and Clinical

Immunology, Charité - University Medicine Berlin,

Berlin, Germany

e-mail: tobias.alexander@ charite.de

\section{B. Sharrack}

Academic Department of Neuroscience and Sheffield NIHR Translational Neuroscience BRC, Sheffield Teaching Hospitals, NHS Foundation Trust, University of Sheffield, Sheffield, UK

\section{Rovira}

BMT Unit, Haematology Department, IDIBAPS, Hospital Clinic, Josep Carreras Leukemia Research Institute (IJC), Barcelona, Spain
}

Initially supported by preclinical animal models and 'serendipitous' case reports, autoHSCT has grown as a promising and feasible treatment option for severe treatment-resistant patients, especially in diseases for which effective therapies are lacking. Allo-HSCT has also been undertaken, although caution related to its intrinsic risks has precluded widespread application.

Following the first international meeting for the use of HSCT in AD in September 1996, the EBMT Autoimmune Diseases Working Party (ADWP) was established in 1997 by Alois Gratwohl, Alberto Marmont, Alan Tyndall and Athanasios Fassas, who developed the registry covering AD indications and produced early guidelines based on consensus opinion (Tyndall and Gratwohl 1997).

\section{Farge}

Unité Clinique de Médecine Interne, Maladies Auto-immunes et Pathologie Vasculaire, UF 04, Hôpital Saint-Louis, EA 3518, AP-HP Assistance Publique des Hôpitaux de Paris, Paris Denis Diderot University, Paris, France

Centre de Référence des Maladies auto-immunes systémiques Rares d'Ile-de-France (site constitutif), Filière FAI2R, Paris, France

\section{J. A. Snowden}

Department of Haematology, Sheffield Teaching Hospitals NHS Foundation Trust, Royal Hallamshire Hospital, Sheffield, UK 
Subsequently the ADWP built productively on these initial achievements, generating studies from the growing registry and developing relationships with other specialist societies, culminating with successful publication of three randomised controlled trials (van Laar et al. 2014; Mancardi et al. 2015; Hawkey et al. 2015) along with updated guidelines for clinical practice (Snowden et al. 2012) and immune monitoring and biobanking (Alexander et al. 2015).

With the increase in evidence, the guidelines have become more disease specific. A successful collaboration involving significant non-European collaborating partners resulted in the EBMT guidelines for auto-HSCT in systemic sclerosis (Farge et al. 2017). A further collaborative review with the European Crohn's and Colitis Organisation (ECCO), which has recently been published, includes recommendations for patient selection, transplant technique and follow-up of HSCT in patients with Crohn's disease (Snowden et al. 2018). Guidelines for MS and neurological diseases are in preparation.

The current state of the EBMT database in relation to various ADs is summarised in Tables 91.1 and 91.2. At the time of writing, over 2500 patients receiving HSCT for an AD have been reported to the EBMT, the largest international database, with activity reported to other registries adding substantially to the worldwide numbers. The most recent EBMT activity survey identified autoimmune diseases as the fastest growing indication group for HSCT (Passweg et al. 2018). Based on this reported activity, this chapter will cover the main neurological, rheumatological and gastroenterological indications for auto-HSCT, along with reference to the rare AD indications and alloge-

Table 91.1 Overview of data reported to the EBMT database (October 2018)

\begin{tabular}{l|l} 
Patients & 2725 \\
\hline Transplant procedures & 2780 \\
\hline Centres/Countries & $267 / 40$ \\
\hline $\begin{array}{l}\text { Autografts/Allografts } \\
\text { Median age at transplantation } \\
\text { (years) }\end{array}$ & $2605(94 \%) / 175(6 \%)$ \\
Male/Female & $37(0.18-76)$ \\
\hline
\end{tabular}

neic HSCT. More detailed literature can be sourced from recently published reviews (Snowden et al. 2017, 2018; Burt and Farge 2018; Alexander and Hiepe 2017).

\subsection{HSCT for Multiple Sclerosis (MS)}

Since the first case report of using auto-HSCT as a treatment for MS was published in 1995, the EBMT registry has now accumulated over 1000 patients (Table 91.2). This treatment was initially used in patients with advanced progressive disease as a rescue therapy with limited efficacy. More recently, its use in patients with active relapsing MS has been associated with prolonged clinical and MRI responses and, in some cases, significant improvement in disability to a degree rarely seen with other disease-modifying drugs (Muraro et al. 2017). Only one randomised controlled phase II trial of auto-HSCT has been reported in the literature (Mancardi et al. 2015). The bulk of the data has been provided by observational cohort studies in which patients failing to respond to standard disease-modifying drugs were treated with HSCT. Burman et al. identified the four most rigorously conducted cohort studies in which a total of 188 relapsing MS patients received auto-HSCT (Burman et al. 2018). In these studies, PFS was observed in $70-91 \%$ of patients at 5 years (where progression is defined as a deterioration of at least $0.5-1$ points from baseline in the Expanded Disability Status Scale or EDSS). Furthermore, no evidence of disease activity (NEDA), defined as the absence of clinical relapses, disability progression, and MRI disease activity, was observed in 70-92\% of patients at 2 years post-transplantation (Burman et al. 2018; Burt et al. 2015; Atkins et al. 2016; Nash et al. 2017).

The conditioning regimens used in MS vary between treatment centres. The balance of efficacy and acceptable safety profile of 'intermediate intensity' regimens, i.e. either the specific 'BEAM + ATG' regimen or the more generic regimen of CY $200 \mathrm{mg} / \mathrm{kg}$ combined with ATG, led to their recommended use in the current 
Table 91.2 Distribution of diagnosis in the EBMT database (October 2018)

\begin{tabular}{|l|r|l|r|}
\hline Multiple sclerosis (MS) & 1285 & Haematological diseases & 113 \\
\hline Connective tissue diseases & 735 & immune thrombocytopenia purpura (ITP) & 33 \\
\hline Systemic sclerosis & 559 & Haemolytic anaemia & 27 \\
\hline Systemic lupus erythemathosus & 117 & Evans syndrome & 25 \\
\hline Polymalgia/dermatomyositis & 18 & Other & 28 \\
\hline Sjögren's syndrome & 5 & Vasculitis & 56 \\
\hline Antiphospholipid syndrome & 6 & Granulomatosis with polyangiitis (GPA) & 14 \\
\hline Other & 30 & Behcet's disease & 11 \\
\hline Arthritis & 186 & Takayasu's arteriitis & 2 \\
\hline Rheumatoid arthritis & 82 & Microscopic polyangiitis (MPA) & 3 \\
\hline Juvenile chronic arthritis (JIA): & & Polyarteritis nodosa (PAN) & 1 \\
\hline - Systemic JIA & 59 & Eosinophilic granulomatosis with polyangiitis (EGPA) (EGPA) & 2 \\
\hline$\bullet$ Other JIA & 18 & Other & 23 \\
\hline • Polyarticular JIA & 17 & Other neurological diseases & 114 \\
\hline Psoriatic arthritis & 3 & Chronic inflammatory demyelinating polyneuropathy (CIDP) & 52 \\
\hline Other & 7 & Neuromyelitis optica & 26 \\
\hline Inflammatory bowel diseases & 220 & Myasthenia gravis & 8 \\
\hline Crohn's disease & 182 & Other & 28 \\
\hline Ulcerative colitis & 4 & Insulin dependent diabetes mellitus (IDDM) & 20 \\
\hline Other & 34 & Other & 51 \\
\hline
\end{tabular}

EBMT ADWP guidelines (Snowden et al. 2012). More recent data suggests that 'high-intensity' regimens, incorporating $\mathrm{BU}$, have higher rates of PFS but potentially greater toxicity, including TRM (Atkins et al. 2016). Retrospective registry data suggest that graft purging has no added benefit to the transplant outcome (Snowden et al. 2012).

Currently, auto-HSCT is considered to be most effective in patients with relapsing MS, who are not older than 45 years, have had the illness for less than 10 years, are not very disabled (EDSS $\leq 6)$ and have very active disease with evidence of enhancement on their MRI (Muraro et al. 2017). An international randomised controlled phase III trial is currently being conducted to compare auto-HSCT with currently available MDTs (MIST Study, ClinicalTrials.gov Identifier: NCT00273364) and is anticipated to report in full in 2021.

\subsection{HSCT for Systemic Sclerosis (SSc)}

Over the past 10 years, results from large European prospective observational studies confirmed that SSc patients benefit only marginally from standard immunosuppressive drugs, including $\mathrm{CY}$, with a progressive increase in $\mathrm{SSc}$ specific mortality, predominantly related to cardiac (31\%) and pulmonary causes (18\%). Indications for auto-HSCT in SSc have increased (Snowden et al. 2018) since three successive randomised trials, namely, ASSIST (Burt et al. 2011), ASTIS (van Laar et al. 2014) and SCOT (Sullivan et al. 2018), have now demonstrated that auto-HSCT is superior to CY for early rapidly progressive SSc in terms of long-term survival as well as improvement of lung function and skin fibrosis.

In addition, patient selection was shown to directly affect transplant outcomes (Farge et al. 2017) with specific concern for cardiac involvement, undetected by echocardiography alone, becoming clinically overt during the transplant procedure, under the stress of fluid overload, CY and ATG administration and sepsis. Current guidelines recommend auto-HSCT for patients with early diffuse SSc with a modified Rodnan skin score $\geq 15$ plus major organ involvement in respiratory, cardiovascular or renal systems and treatment should be performed in JACIEaccredited centres where combined expertise from SSc disease specialist and dedicated transplant team can assess and follow patients before, 
during and after the procedure according to Good Clinical Practice (Snowden et al. 2018). Since toxicity and efficacy arise from individual patient selection and the conditioning regimen, different chemotherapies may account for subtle differences in results, and further studies are warranted to analyse the use of attenuated conditioning regimen, according to cardiac function and risk for renal crises (Burt and Farge 2018).

\subsection{HSCT for Systemic Lupus Erythematosus (SLE)}

Following the first transplant performed in 1996 by Alberto Marmont and colleagues, several phase I/phase II clinical trials have been reported covering approximately 300 SLE patients worldwide to date (Alexander and Hiepe 2017). The two largest experiences on auto-HSCT for SLE so far come from EBMT data registry ( $n=53$; mean follow-up, 25 months) and from the singlecentre trial by Northwestern University ( $n=50$; mean follow-up, 29 months), both demonstrating a probability of 5-year DFS of 50\% despite discontinuation of chronic IS. Subsequently, a follow-up study from the EBMT registry reported the outcome of auto-HSCT in SLE with various regimens between 2001 and $2008(n=28$; median follow-up, 38 months; range, 1-110 months) (Alchi et al. 2013). Although PFS in this study was only $29 \%$ at 5 years, TRM had gradually improved. In addition, this study indicated that CD34-selection was associated with a significantly reduced relapse incidence.

More recently, reports from two independent Chinese groups, both with 10-year follow-up, demonstrated remarkable clinical responses with PFS of $86 \%$ and $68 \%$, respectively, while TRM across both studies was only $2 \%$. Current recommendations suggest HSCT in patients with sustained or relapsed active disease (BILAG category A) remaining steroid dependent after at least 6 months of the best standard therapy (including MMF and CY with or without anti-CD20), with documented evidence of visceral involvement (Snowden et al. 2012). The only controlled study conducted to compare auto-HSCT with cur- rently available MDTs in SLE is currently ongoing in a phase II multicentre trial in Germany (ClinicalTrials.gov Identifier: NCT00750971).

\subsection{HSCT for Crohn's Disease (CD)}

Despite the major recent progress in the treatment of CD, based around corticosteroids, IS (thiopurines, MTX) and biologic therapies (Ab targeting TNF $\alpha, \alpha 4 \beta 7$ integrin or IL-12/IL-23), some patients fail all available therapies. In many cases, surgery may be an option but may lead to short bowel syndrome or to a definitive stoma, which may be unacceptable to patients. With this background, in the past few years, auto-HSCT has emerged as a promising therapy in a subset of patients in whom the disease is refractory to all available therapies, with progressive tissue damage and potentially reduced life expectancy.

Auto-HSCT has been investigated in several studies with encouraging responses, some prolonged, although a progressive incidence of relapse with long-term follow-up is recognised (Burt et al. 2010; Lopez-Garcia et al. 2017). Furthermore, patients regain response to antiTNF therapy although they had been refractory to this drug class prior to HSCT.

In Europe, the EBMT sponsored ASTIC trial in patients with refractory $\mathrm{CD}$ and produced apparently negative results as few patients after auto-HSCT met the stringent primary composite endpoint of clinical remission for 3 months (Hawkey et al. 2015). However, it should not be assumed that this single trial provides the definitive answer to the benefit of auto-HSCT in CD, as the number of patients included was limited and encouraging long-term follow-up of ASTIC trial patients has since been reported (Lindsay et al. 2017). Encouraging results have also been reported by an EBMT retrospective analysis of 82 treatment-resistant patients who were not in the ASTIC trial. In this difficult-to-treat group of patients, around a quarter maintained remission without further medical therapy, and long-term disease control was maintained with reintroduction of salvage therapies in the major- 
ity of patients who relapsed. TRM occurred in one patient (Brierley 2018).

Recently, the EBMT ADWP and ECCO (European Crohn's and Colitis Organisation) have published a joint position paper of auto-HSCT in $\mathrm{CD}$, which recommends auto-HSCT ideally in the context of a multicentre clinical trial but on an individual basis may currently be considered for patients with active CD refractory to IS and biological treatments or unacceptable risks of surgical management (Snowden et al. 2018).

\subsection{Allogeneic HSCT for Autoimmune Diseases}

Autoimmune diseases have also been treated with allo-HSCT from MRD, URD and CB sources. In the last 20 years, the EBMT registry has collated 165 cases, representing just $6 \%$ of the total. Because of the higher procedural risks and potential long-term impact on quality of life from late effects, allo-HSCT has been largely restricted to life-threatening $\mathrm{AD}$ in paediatric practice with the most common indications in immune cytopenia followed by arthritis (Snowden et al. 2017). In a recent summary of cases in the registry between 1997 and 2015, 105 patients undergoing allo-HSCT were treated with conditioning regimens. The median age was 12 years (range 1-62). Outcome following first allo-HSCT included 3- and 5-year OS of $67 \%$ and $64 \%$, PFS $59 \%$ and $56 \%$ and incidence of relapse $21 \%$ and $24 \%$, respectively. Compared with auto-HSCT, NRM is relatively high at $13 \%$ at 100 days, although this plateaus at $20 \%$ at 3 and 5 years.

In summary, experience in allo-HSCT is limited, but long-term data is supportive of basic biological differences in the responses of $\mathrm{AD}$ to allo- and auto-HSCT in terms of the ability to cure. However, with the risks NRM of around $20 \%$, allo-HSCT will remain limited and developmental in its application to AD. With time, allo-HSCT in AD has become safer, perhaps with better patient selection, and activity continues, particularly in the paediatric field. In addition, there has been increasing recognition that the nature of the genetic component of some $\mathrm{AD}$ means that allo-HSCT is the only realistic approach for long-term disease control. Thus there is renewed interest in this area, particularly where there is overlap with autoinflammatory and immunodeficiency diseases.

\subsection{Other Indications}

A variety of other ADs have been treated (Table 91.2). Haematological immune cytopenias have been treated with a mixture of auto-HSCT and allo-HSCT. Type 1 diabetes in early 'honeymoon' phase has been the subject of clinical trials, with some ability to prevent or reduce insulin requirements. Otherwise there is a mixture of rarer neurological, rheumatological and gastroenterological indications for which the registry is essential for developing an evidence base. Cautious recommendations for these rare indications are provided in the EBMT ADWP guidelines (Snowden et al. 2012).

\subsection{Mechanisms of Action}

HSCT represented an opportunity to gain insights into the aetiology and pathogenesis of AD. Through destroying a dysfunctional autoreactive immune system and rebuilding it with auto-HSCT, it has been possible to demonstrate immune 'rebooting' that can occur through thymic reactivation and re-diversification and other changes in T-cell and B-cell repertoire and regulatory cell function in various disease settings. Allo-HSCT has been less well explored, although there is an element of immune replacement and evidence for a 'graft-versus-autoimmune effect' (Alexander et al. 2016).

\subsection{Conclusions and Future Directions}

With accumulating evidence and improved outcomes along with recognition that modern biological and other therapies are not universally effective (Snowden et al. 2017), ADs have 
become the fastest-growing indication for HSCT (Passweg et al. 2018). Initially applied as salvage therapy in patients with poor prognosis, HSCT has emerged as a promising treatment option for AD patients earlier in the treatment algorithm. This is the result of positive results from large phase II and randomised controlled phase III trials and updated guidelines for patient selection and transplant technique (Snowden et al. 2012, 2018; Farge et al. 2017). In 2018, the major indications for HSCT for AD are MS, SSc and CD for which significant subsets of patients still show an unsatisfactory response to both conventional and targeted biologic therapies.

Moving forward, further efforts are needed to drive HSCT into routine clinical care. It is recommended that patients should be treated in experienced and JACIE-accredited transplant centres in a multidisciplinary setting. A future goal is to optimise the conditioning regimens according to disease-specific requirements and to outbalance the intensity to maintain outcomes while minimising toxicity and TRM risk. In addition, comprehensive data reporting, harmonisation and exploitation of existing biobanking infrastructure (Alexander et al. 2015), education at individual centre and network level and health economic evaluations along with evidence-based recommendations will establish the future place of HSCT in the treatment algorithms for various autoimmune and inflammatory diseases.

Acknowledgement The authors thank Manuela Badoglio for helpful assistance with data management.

\section{Key Points}

- With accumulating evidence, including randomised controlled trials, AD has become the fastest-growing indication for HSCT.

- Major indications for HSCT in AD include multiple sclerosis (MS), systemic sclerosis (SSc) and Crohn's disease (CD), where auto-HSCT is now featuring in treatment algorithms.

- Although HSCT for ADs is predominantly autologous, there is renewed interest in allo-HSCT, particularly in ADs with autoinflammatory and immunodeficiency components.

- It is recommended that all patients should be treated in experienced and JACIE-accredited transplant centres with close multidisciplinary collaboration and reporting of data to the EBMT registry.

- Immune reconstitution studies are providing insights into the mechanisms of immune reset following HSCT and disease processes underlying various autoimmune diseases.

\section{References}

Alchi B, Jayne D, Labopin M, et al. Autologous haematopoietic stem cell transplantation for systemic lupus erythematosus: data from the European Group for Blood and Marrow Transplantation registry. Lupus. 2013;22:245-53.

Alexander T, Hiepe F. Autologous haematopoietic stem cell transplantation for systemic lupus erythematosus: time ready for a paradigm shift? Clin Exp Rheumatol. 2017;35:359-61.

Alexander T, Bondanza A, Muraro PA, et al. SCT for severe autoimmune diseases: consensus guidelines of the European Society for Blood and Marrow Transplantation for immune monitoring and biobanking. Bone Marrow Transplant. 2015;50:173-80.

Alexander T, Arnold R, Hiepe F, RAIDbruch A. Resetting the immune system with immunoablation and autologous haematopoietic stem cell transplantation in autoimmune diseases. Clin Exp Rheumatol. 2016;34:53-7.

Atkins HL, Bowman M, Allan D, et al. Immunoablation and autologous haemopoietic stem-cell transplantation for aggressive multiple sclerosis: a multicentre single-group phase 2 trial. Lancet. 2016;388:576-85.

Burman J, Tolf A, Hagglund H, Askmark H. Autologous haematopoietic stem cell transplantation for neurological diseases. J Neurol Neurosurg Psychiatry. 2018;89:147-55.

Burt RK, Farge D. Systemic sclerosis: autologous HSCT is efficacious, but can we make it safer? Nat Rev Rheumatol. 2018;14:189-91.

Burt RK, Craig RM, Milanetti F, et al. Autologous nonmyeloablative hematopoietic stem cell transplantation in patients with severe anti-TNF refractory Crohn disease: long-term follow-up. Blood. 2010;116:6123-32.

Burt RK, Shah SJ, Dill K, et al. Autologous nonmyeloablative haemopoietic stem-cell transplanta- 
tion compared with pulse cyclophosphamide once per month for systemic sclerosis (ASSIST): an open-label, randomised phase 2 trial. Lancet. 2011;378:498-506.

Burt RK, Balabanov R, Han X, et al. Association of nonmyeloablative hematopoietic stem cell transplantation with neurological disability in patients with relapsingremitting multiple sclerosis. JAMA. 2015;313:275-84.

Brierley CK et al, J Crohns Colitis. 2018. https://doi.org/10.1093/ecco-jcc/jjy069.

Farge D, Burt RK, Oliveira MC, et al. Cardiopulmonary assessment of patients with systemic sclerosis for hematopoietic stem cell transplantation: recommendations from the European Society for Blood and Marrow Transplantation Autoimmune Diseases Working Party and collaborating partners. Bone Marrow Transplant. 2017;52:1495-503.

Hawkey CJ, Allez M, Clark MM, et al. Autologous hematopoetic stem cell transplantation for refractory Crohn disease: a randomized clinical trial. JAMA. 2015;314:2524-34.

Lindsay JO, Allez M, Clark M, et al. Autologous stemcell transplantation in treatment-refractory Crohn's disease: an analysis of pooled data from the ASTIC trial. Lancet Gastroenterol Hepatol. 2017;2:399-406.

Lopez-Garcia A, Rovira M, Jauregui-Amezaga A, et al. Autologous hematopoietic stem cell transplantation for refractory Crohn's disease: efficacy in a singlecentre cohort. J Crohns Colitis. 2017;11:1161-8.

Mancardi GL, Sormani MP, Gualandi F, et al. Autologous hematopoietic stem cell transplantation in multiple sclerosis: a phase II trial. Neurology. 2015;84:981-8.

Muraro PA, Pasquini M, Atkins HL, et al. Long-term outcomes after autologous hematopoietic stem cell transplantation for multiple sclerosis. JAMA Neurol. 2017;74:459-69.

Nash RA, Hutton GJ, Racke MK, et al. High-dose immunosuppressive therapy and autologous HCT for relapsing-remitting MS. Neurology. 2017;88:842-52.
Passweg JR, Baldomero H, BAIDer P, et al. Is the use of unrelated donor transplantation leveling off in Europe? The 2016 European Society for Blood and Marrow Transplant activity survey report. Bone Marrow Transplant. 2018. https://doi. org/10.1038/s41409-018-0153-1. [Epub aheAID of print].

Snowden JA, Saccardi R, Allez M, et al. Haematopoietic SCT in severe autoimmune diseases: updated guidelines of the European Group for Blood and Marrow Transplantation. Bone Marrow Transplant. 2012;47:770-90.

Snowden JA, Badoglio M, Labopin M, et al. Evolution, trends, outcomes, and economics of hematopoietic stem cell transplantation in severe autoimmune diseases. Blood Adv. 2017;1:2742-55.

Snowden JA, Panes J, Alexander T, et al. Autologous haematopoietic stem cell transplantation (AHSCT) in severe Crohn's disease: a review on behalf of ECCO and EBMT. J Crohns Colitis. 2018;12: 476-88.

Sullivan KM, Goldmuntz EA, Keyes-Elstein L, et al. Myeloablative autologous stem-cell transplantation for severe scleroderma. N Engl J Med. 2018;378: $35-47$.

Tyndall A, Gratwohl A. Blood and marrow stem cell transplants in auto-immune disease: a consensus report written on behalf of the European League against Rheumatism (EULAR) and the European Group for Blood and Marrow Transplantation (EBMT). Bone Marrow Transplant. 1997;19:643-5.

van Laar JM, Farge D, Sont JK, et al. Autologous hematopoietic stem cell transplantation vs intravenous pulse cyclophosphamide in diffuse cutaneous systemic sclerosis: a randomized clinical trial. JAMA. 2014;311:2490-8.

Open Access This chapter is licensed under the terms of the Creative Commons Attribution 4.0 International License (http://creativecommons.org/licenses/by/4.0/), which permits use, sharing, adaptation, distribution and reproduction in any medium or format, as long as you give appropriate credit to the original author(s) and the source, provide a link to the Creative Commons license and indicate if changes were made.

The images or other third party material in this chapter are included in the chapter's Creative Commons license, unless indicated otherwise in a credit line to the material. If material is not included in the chapter's Creative Commons license and your intended use is not permitted by statutory regulation or exceeds the permitted use, you will need to obtain permission directly from the copyright holder.

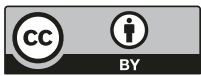

